\title{
Editorial: Population Pharmacogenomics (PGx): From Variant Identification to Clinical Implementation
}

\author{
Masahiro Hiratsuka ${ }^{1}$, Yitian Zhou ${ }^{2}$ and Volker M. Lauschke ${ }^{2,3 *}$ \\ ${ }^{1}$ Graduate School of Pharmaceutical Sciences, Tohoku University, Sendai, Japan, ${ }^{2}$ Department of Physiology and \\ Pharmacology, Karolinska Institutet, Stockholm, Sweden, ${ }^{3}$ Dr. Margarete Fischer-Bosch Institute of Clinical Pharmacology, \\ Stuttgart, Germany
}

Keywords: population genetics, pharmacogenomics (PGx), precision medicine, CYP, N-acetyltransferase (NAT)

\section{Editorial on the Research Topic}

Population Pharmacogenomics (PGx): From Variant Identification to Clinical Implementation

It is by now well-established that genetic differences underlie the inter-individual variability in pharmacokinetics, response, and toxicity of many drugs. Gene families of particular interest in this context include those encoding cytochrome P450 enzymes (CYPs), other drug metabolizing enzymes, such as N-acetyltransferases (NATs), DPD, and TPMT, as well as drug transporters of the ATP-binding cassette (ABC) and solute carrier (SLC) superfamilies (Lauschke et al., 2017, 2019; Roden et al., 2019). In total, associations between germline polymorphisms and drug-related phenotypes are established for more than 200 drugs and have been included into the respective labels. Well-established and mechanistically understood examples include links between DPYD and TPMT genotype with fluoropyrimidine and thiopurine toxicity, respectively, associations of CYP2D6 and CYP2C19 metabolizer status with the response to various anti-depressants and antipsychotics, as well as correlations between variations in human leukocyte antigen (HLA) genes encoding the major histocompatibility complex and severe hypersensitivity reactions to abacavir, carbamazepine, and allopurinol.

To render the implementation of the testing of such pharmacogenomic biomarkers into routine clinical care a cost-effective allocation of health care resources, it is important to know, besides other parameters, the population-specific frequency of the polymorphisms in question. For instance, previous research showed that preemptive testing of $H L A-B^{*}$ 15:02 of 50-150 patients was sufficient to prevent one adverse drug reaction (ADR) due to carbamazepine in China and South-East Asia, whereas $>10,000$ individuals would need to be tested in Japan, or throughout Africa and Europe (Zhou et al., 2021). As a consequence, preemptive HLA-B*15:02 genotyping is only cost-effective for individuals of South-East Asian ancestry. Similarly, we and others have shown striking ethnogeographic differences for multiple polymorphisms in CYPs, drug transporters, DPYD, and TPMT (Gordon et al., 2014; Fujikura et al., 2015; Mizzi et al., 2016; Zhou et al., 2017, 2020; Schaller and Lauschke, 2019; Petrovic et al., 2020; Xiao et al., 2020; Runcharoen et al., 2021). While these studies provided an important first step, they only considered seven global populations. Thus, further efforts which map the relevant pharmacogenomic variability with higher population resolution can be expected to facilitate the guidance of refined strategies to guide genotype-informed care.

In this Research Topic, Fukunaga et al. mapped the population-specific frequencies of NAT2 alleles and experimentally characterize their functional consequences. Specifically, the authors 
analyzed the frequencies of $N A T 2 * 4, * 5, * 6$, and $* 7$ based on genetic data from 990 Japanese individuals and compared results to available frequency information from the 1000 Genomes Project populations. Furthermore, they experimentally determined Km, Vmax, and CLint of these alleles using eight different model substrates. Based on these data, the authors concluded that frequencies of slow or ultra-slow acetylators, i.e., those carrying one or more ${ }^{*} 5,{ }^{*} 6$, or $* 7$ alleles, was between 30 and 55\% in Europeans, Africans and South Asians, whereas the prevalence of slow acetylator phenotypes in Japanese and other East Asians was substantially lower (4-11\%).

In an additional study, Zhang et al. analyzed the patient benefits of the implementation of CYP2C19 genotyping for the guidance of antiplatelet therapy in China. In this observational study of patients undergoing percutaneous coronary intervention, clopidogrel, or ticagrelor was recommended to be prescribed depending on the absence or presence of CYP2C19 loss-of-function (LOF) alleles $*^{*} 2$ or *3), respectively. While cardiologists mostly adhered to the pharmacogenetic recommendations, those patients with CYP2C19 LOF alleles that were prescribed clopidogrel in opposition to the pharmacogenetic recommendation had significantly higher rates of major cardiac or cerebrovascular adverse events ( 7.8 vs. $4.0 \% ; p=0.029$ ). No significant differences in major bleeding events were observed between genotype and treatment groups. These results are particularly important as the currently available evidence regarding the benefits of pharmacogenomics-guided treatment for cardiovascular diseases is limited with mixed results (Zhu et al., 2020).

Lastly, an interesting study by the Human Heredity and Health in Africa (H3Africa) Consortium provides an overview of the pharmacogenomic variation in Sub-Saharan Africa based on 458 high-coverage whole genome sequences. The authors find drastic differences in population frequencies between the different ethnogeographic groups and identify

\section{REFERENCES}

Fujikura, K., Ingelman-Sundberg, M., and Lauschke, V. M. (2015). Genetic variation in the human cytochrome P450 supergene family. Pharmacogenet. Genomics 25, 584-594. doi: 10.1097/FPC.00000000000 00172

Gordon, A. S., Tabor, H. K., Johnson, A. D., Snively, B. M., Assimes, T. L., Auer, P. L., et al. (2014). Quantifying rare, deleterious variation in 12 human cytochrome P450 drug-metabolism genes in a large-scale exome dataset. Hum. Mol. Genet. 23, 1957-1963. doi: 10.1093/hmg/ ddt588

Lauschke, V. M., Milani, L., and Ingelman-Sundberg, M. (2017). Pharmacogenomic biomarkers for improved drug therapy-recent progress and future developments. AAPS J. 20:4. doi: 10.1208/s12248-0170161-x

Lauschke, V. M., Zhou, Y., and Ingelman-Sundberg, M. (2019). Novel genetic and epigenetic factors of importance for inter-individual differences in drug disposition, response and toxicity. Pharmacol. Ther. 197, 122-152. doi: $10.1016 /$ j.pharmthera.2019.01.002

Mizzi, C., Dalabira, E., Kumuthini, J., Dzimiri, N., Balogh, I., Başak, N., et al. (2016). A European spectrum of pharmacogenomic biomarkers: implications for clinical pharmacogenomics. PLoS ONE 11:e0162866. doi: 10.1371/journal.pone.0162866
930 single nucleotide variants (SNVs) with putative functional consequences, most of which were restricted to specific populations. Together with other studies (Radouani et al., 2020; Pernaute-Lau et al., 2021), this resource increases the available information about the pharmacogenetic diversity in Africa considerably and incentivizes functional testing of the identified variants in question.

In summary, we are confident that the papers included in this Research Topic increase our understanding of pharmacogenomic population diversity and provide useful information for the optimization and facilitation of population-specific precision public health efforts in previously understudied populations.

\section{AUTHOR CONTRIBUTIONS}

All authors contributed to the writing and approved the final version of the manuscript.

\section{FUNDING}

MH was supported by grants from the Japan Agency for Medical Research and Development (AMED) (Grant number JP19kk0305009). VL receives support from the Swedish Research Council (grant agreement numbers: 2016-01153, 2016-01154, and 2019-01837), by the EU/EFPIA/OICR/McGill/KTH/Diamond Innovative Medicines Initiative 2 Joint Undertaking (EUbOPEN grant number 875510), by the Swedish Strategic Research Programmes in Diabetes (SFO Diabetes) and Stem Cells and Regenerative Medicine (SFO StratRegen), as well as by the European Union'Horizon 2020 research and innovation program UPGx (grant agreement number 668353). Furthermore, VL acknowledges support from Merck KGaA, Eli Lilly and Company, and by the Robert Bosch Foundation.
Pernaute-Lau, L., Adegnika, A. A., Zhou, Y., Zinsou, J. F., Gil, J. P., Krishna, S., et al. (2021). Pharmacogene sequencing of a gabonese population with severe plasmodium falciparum malaria reveals multiple novel variants with putative relevance for antimalarial treatment. Antimicrob. Agents Chemother. 65:e027521. doi: 10.1128/AAC.00275-21

Petrovic, J., Pesic, V., and Lauschke, V. M. (2020). Frequencies of clinically important CYP2C19 and CYP2D6 alleles are graded across Europe. Eur. J. Hum. Genet. 28, 88-94. doi: 10.1038/s41431-019-0480-8

Radouani, F., Zass, L., Hamdi, Y., Rocha, J. D., Sallam, R., Abdelhak, S., et al. (2020). A review of clinical pharmacogenetics Studies in African populations. Per. Med. 17, 155-170. doi: 10.2217/pme-2019-0110

Roden, D. M., McLeod, H. L., Relling, M. V., Williams, M. S., Mensah, G. A., Peterson, J. F., et al. (2019). Pharmacogenomics. Lancet 394, 521-532. doi: 10.1016/S0140-6736(19)31276-0

Runcharoen, C., Fukunaga, K., Sensorn, I., Iemwimangsa, N., Klumsathian, S., Tong, H., et al. (2021). Prevalence of pharmacogenomic variants in 100 pharmacogenes among Southeast Asian populations under the collaboration of the Southeast Asian Pharmacogenomics Research Network (SEAPharm). Hum. Genome Var. 8:7. doi: 10.1038/s41439-021-00 $135-\mathrm{Z}$

Schaller, L., and Lauschke, V. M. (2019). The genetic landscape of the human solute carrier (SLC) transporter superfamily. Hum. Genet. 138, 1359-1377. doi: 10.1007/s00439-019-02081-x 
Xiao, Q., Zhou, Y., and Lauschke, V. M. (2020). Ethnogeographic and interindividual variability of human ABC transporters. Hum. Genet. 139, 623-646. doi: 10.1007/s00439-020-02150-6

Zhou, Y., Dagli Hernandez, C., and Lauschke, V. M. (2020). Populationscale predictions of DPD and TPMT phenotypes using a quantitative pharmacogene-specific ensemble classifier. Br. J. Cancer 123, 1782-1789. doi: 10.1038/s41416-020-01084-0

Zhou, Y., Ingelman-Sundberg, M., and Lauschke, V. M. (2017). Worldwide distribution of cytochrome P450 alleles: a meta-analysis of population-scale sequencing projects. Clin. Pharmacol. Ther. 102, 688-700. doi: 10.1002/ cpt. 690

Zhou, Y., Krebs, K., Milani, L., and Lauschke, V. M. (2021). Global frequencies of clinically important HLA alleles and their implications for the cost-effectiveness of preemptive pharmacogenetic testing. Clin. Pharmacol. Ther. 109, 160-174. doi: 10.1002/cp t.1944

Zhu, Y., Swanson, K. M., Rojas, R. L., Wang, Z., St Stauver, J. L., Visscher, S. L., et al. (2020). Systematic review of the evidence on the cost-effectiveness of pharmacogenomics-guided treatment for cardiovascular diseases. Genet. Med. 22, 475-486. doi: 10.1038/s41436-0190667-y
Conflict of Interest: YZ and VL are co-founders and shareholders of PersoMedix $\mathrm{AB}$. In addition, $\mathrm{VL}$ is $\mathrm{CEO}$ and shareholder of HepaPredict $\mathrm{AB}$ and discloses consultancy work for Enginzyme AB.

The remaining author declares that the research was conducted in the absence of any commercial or financial relationships that could be construed as a potential conflict of interest.

Publisher's Note: All claims expressed in this article are solely those of the authors and do not necessarily represent those of their affiliated organizations, or those of the publisher, the editors and the reviewers. Any product that may be evaluated in this article, or claim that may be made by its manufacturer, is not guaranteed or endorsed by the publisher.

Copyright (c) 2021 Hiratsuka, Zhou and Lauschke. This is an open-access article distributed under the terms of the Creative Commons Attribution License (CC BY).

The use, distribution or reproduction in other forums is permitted, provided the original author(s) and the copyright owner(s) are credited and that the original publication in this journal is cited, in accordance with accepted academic practice. No use, distribution or reproduction is permitted which does not comply with these terms. 